\title{
Assessing the hodgepodge of non-mapped reads in bacterial transcriptomes: real or artifactual RNA chimeras?
}

Verónica Lloréns-Rico ${ }^{1,2}$, Luis Serrano ${ }^{1,2,3^{*}}$ and Maria Lluch-Senar ${ }^{1,2^{*}}$

\begin{abstract}
Background: RNA sequencing methods have already altered our view of the extent and complexity of bacterial and eukaryotic transcriptomes, revealing rare transcript isoforms (circular RNAs, RNA chimeras) that could play an important role in their biology.

Results: We performed an analysis of chimera formation by four different computational approaches, including a custom designed pipeline, to study the transcriptomes of $M$. pneumoniae and $P$. aeruginosa, as well as mixtures of both. We found that rare transcript isoforms detected by conventional pipelines of analysis could be artifacts of the experimental procedure used in the library preparation, and that they are protocol-dependent.

Conclusion: By using a customized pipeline we show that optimal library preparation protocol and the pipeline to analyze the results are crucial to identify real chimeric RNAs.
\end{abstract}

Keywords: Chimeric RNAs, Fusion transcripts, RNA-seq, Library preparation protocols

\section{Background}

In recent years, different groups have shown the existence of RNA editing in different species [1-3], and more recently the possibility of the presence of the so-called RNA chimeras $[4,5]$. These are transcripts that contain fragments from independent RNA molecules. RNA chimeras may arise from chromosome translocations, as found in eukaryotes, where they often play a role as transforming oncogenes [6-8]. Furthermore, mRNA chimeras can also arise as product of transcriptional or post-transcriptional events. Examples in the archaeal domain are tRNAs that are composed of pieces transcribed from different regions of the genome [9-12]. Also in eukaryotes, several reports have suggested the presence of RNA chimeras that are not the result of chromosome rearrangement in eukaryotes [13,14]. This implies the existence of mechanisms for end joining of independent RNA transcripts and, if true, could point out to new hidden complexity. Spliceosome-mediated trans-splicing has been suggested

\footnotetext{
*Correspondence: luis.serrano@crg.eu; maria.lluch@crg.eu

${ }^{1}$ EMBL/CRG Systems Biology Research Unit, Centre for Genomic Regulation (CRG), Dr. Aiguader 88, 08003 Barcelona, Spain

${ }^{2}$ Universitat Pompeu Fabra (UPF), Dr. Aiguader 88, 08003 Barcelona, Spain

Full list of author information is available at the end of the article
}

to be one possible mechanism for RNA chimera formation in eukaryotes [13]. Another proposed mechanism for the formation of fusion transcripts is intergenic splicing. This occurs when transcription fails to terminate at a transcription termination site and therefore the nascent transcript is extended until the termination site of the next gene. The resulting bicistronic transcript is processed to obtain a mature RNA formed by exons from two adjacent genes, and these chimeras are often regarded as readthrough chimeras or transcription-induced chimeras $[15,16]$. Some recent studies have shown that these chimeras that do not result from chromosomal translocations are translated and may encode for novel functions inside the cell $[17,18]$.

Several computational approaches have been developed for the detection of chimeric transcripts in RNA-seq data [19-26]. Although all of these computational methods show good sensitivity, true identification of chimeric transcripts could be hampered by experimental artifacts. Indeed, there are reports that indicate that artifactual chimeras could be generated in the RNA sample preparation for deep-sequencing $[27,28]$. Multiple factors, including pairwise sequence identity between rRNA genes, number of PCR cycles, and relative abundance of genespecific PCR templates have been shown to influence 
chimera formation [27]. Furthermore, the rapid changes in these cutting-edge technologies demand fast adaptation, limiting the time for proper optimization of library preparation protocols, as well as of the software tools used to analyze the results. Thus, it is important to carefully select the mapping software according to the structure of the RNA-seq data under analysis. Some algorithms, as FusionMap [20], ChimeraScan [25] and TopHat Fusion [26] apply different filters in order to validate real chimeric RNAs. For instance, TopHat Fusion [26] considers the total number of reads that cover a given fusion transcript and the read distribution around the chimeric junction, as well as the number of unique reads spanning each junction. It also applies different filters for RNA polymerase read-through (given leaky termination sites, the RNA polymerase would be able to continue transcribing neighbor genes) and minimum anchor length at each side of the junction. ChimeraScan [25] introduces other filters such as the PE mates distance regarding the junction site, and the isoform fraction of the chimera respect to the genes that are forming it. FusionMap [20] also considers read quality in order to discard artifactual chimeras arising from read mismapping.

Here, we want to determine if prokaryotic RNA chimeras exist and to what extent they may be the result of artifact generation both in the library preparation protocols and the analysis methods. To do so we chose two bacteria belonging to gram negative (Pseudomonas aeruginosa) and gram positive (Mycoplasma pneumoniae) classes. Both genomes are fully sequenced $[29,30]$ and in the case of $M$. pneumoniae, aside from being very small $(816 \mathrm{~Kb})$ a genome-wide transcriptome analysis is available [31]. To control for potential artifacts in sample preparation we mixed $P$. aeruginosa and $M$. pneumoniae at different stages of the RNA extraction and preparation, and performed RNA-seq of the isolated and mixed samples. We analyzed two widely used protocols for library preparation (Directional RNA-seq (TruSeq small RNA Sample Prep Kit, Illumina) and TruSeq stranded RNAseq (TruSeq Stranded mRNA Sample Prep Kit, Illumina), see Methods) to study the impact of these protocols on chimera formation. Both protocols are widely used in the analysis of eukaryotic and prokaryotic transcriptomes. Whilst the first one is mainly used for small RNAs in eukaryotes, such as siRNAs or microRNAs, the second is mostly used for mRNA sequencing [32-35]. Regarding bacteria, both methods have been used for total RNA library preparation [36,37]. To analyze the data, we used FusionMap [20], ChimeraScan [25] and TopHat-Fusion [26] (see Additional file 1: Figure S1) as well as a customized pipeline designed to filter out artifacts.

Our analysis detected large numbers of putative chimeric RNAs that were revealed to be artifacts generated during library preparation protocols, using widely accepted sequencing methodologies. Also, we found that the methodology used for sequence analysis could result in different non-real chimeras identified. Therefore, care should be taken with the selection of a sequencing protocol and software for the analysis when annotating fusion transcripts in different organisms.

\section{Results and discussion \\ Sequence mapping}

To analyze the origin of chimeric RNAs and to discern if they are artifacts derived of library preparation or natural chimeric RNAs, we prepared different samples by mixing RNA of $M$. pneumoniae and P. aeruginosa at different ratios $(1: 1 ; 1: 5$ and 1:50), as well as non-mixed samples. Mixing was done immediately after RNA extraction. Another sample was obtained by mixing the cells of both bacteria prior to RNA extraction (Cell Mix). Libraries were prepared using the Directional RNA-seq library protocol (see Methods). We used two alternative short read mappers: GEM-mapper and Bowtie [38,39]. With GEM-mapper the sequences were mapped individually as single-end (SE) reads without considering the associated mates, while Bowtie was used to map paired-end (PE) mates and calculate the insert lengths in between both mates (Figure 1). In both cases it was requested that the reads mapped uniquely to a single position in the genome. It has been previously reported that in PE sequencing a small percentage of second mate reads are not correctly assigned to their corresponding first mate read. This will lead to the obtaining of artifactual chimeras [40]. In contrast to the previous methods, we used SE reads in the first step of the analysis to identify the junction of putative chimeric RNAs. Then, we applied PE mapping to validate the candidates for the study of putative chimeric RNAs in all the samples previously described, by supporting the reads that spanned the chimeric RNA junction. Both SE and $P E$ reads were used to validate the chimeric candidates, and several filtering steps were applied in order to discard potential artifacts arising from the different steps of library preparation protocols.

Deep sequencing analysis of $M$. pneumoniae and $P$. aeruginosa samples in the RNA mix samples showed a significant number of chimeric candidates (33389 in the 1:1 ratio RNA mix sample), the majority of them involving tRNAs and rRNAs in both species (for instance, in the $1: 1$ ratio RNA mix sample, $70.6 \%$ of the chimeras were formed by two rRNAs, and $0.96 \%$ were formed by two tRNAs, see Additional file 1: Figure S1), as well as a significant proportion of circular RNAs $(10.8 \%$ of the rare isoforms observed in the 1:1 ratio RNA mix sample corresponded to putative circular RNAs, $99.3 \%$ of them were formed in rRNAs, the remaining $0.7 \%$ correspond to circular RNAs formed by tRNAs; Additional file 1: Figure S1). We also identified a small percentage $(0.02 \%)$ of chimeric 


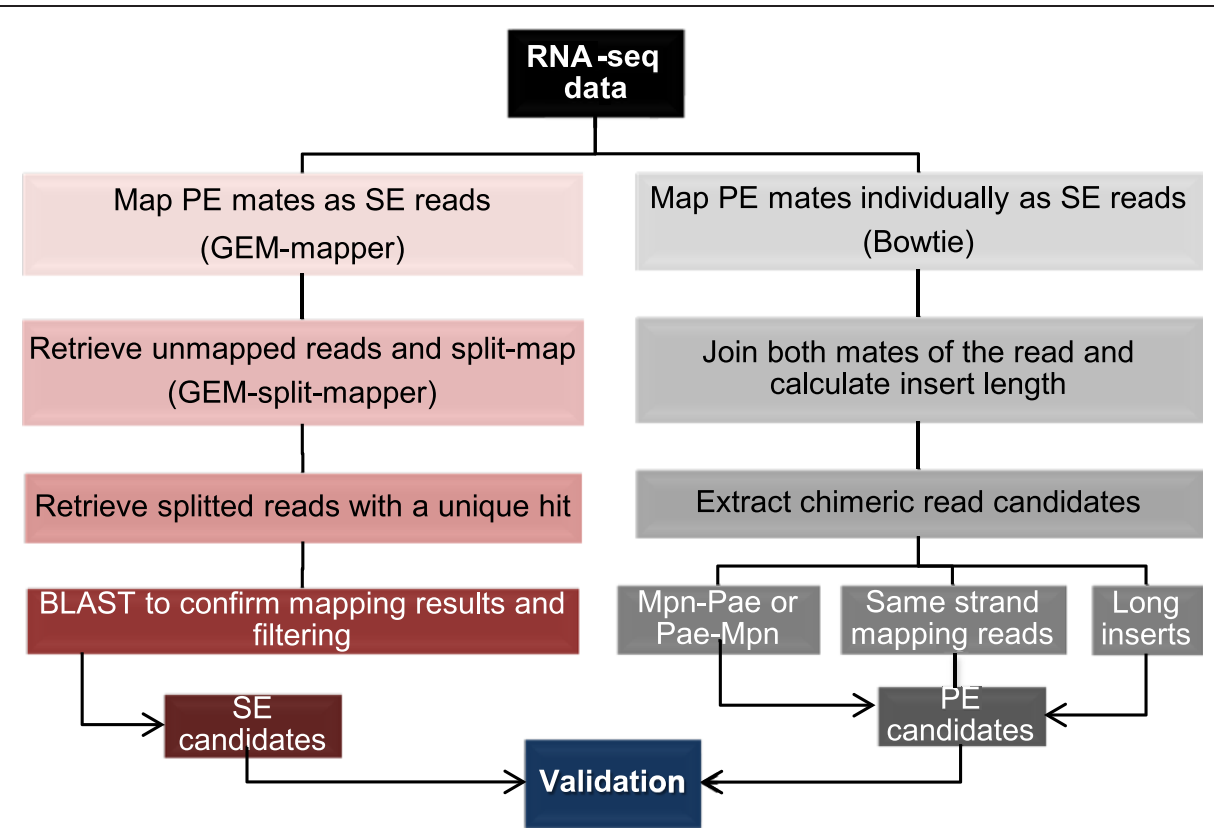

Figure 1 Pipeline of the RNAseq data analysis. Two complementary approaches have been integrated to analyze the putative chimeric RNAs: i) mapping pair end mates as single end by using GEM-mapper and ii) mapping pair ends individually as single ends using Bowtie. After the two complementary mapping strategies there is a validation procedure that integrates three steps: i) determination of confidential scores that consider the levels of expression of the genes that originate the chimeric RNA; ii) filter by unique reads that considers the number of different reads that represent the chimeric junction and iii) filter by staircase profile that select for those chimeric variants that show a homogenous distribution of counts for the different reads that cover the junction.

mRNAs. However, we found that the second ORF was kept in frame in around $33 \%$ of the mRNA chimeras, as expected by chance, for inter- and intra-species mRNA chimeras, thus indicating random fusion events.

In the RNA mix experiment at a ratio $1: 1$ we found around $30 \%$ of M. pneumoniae chimeras, $50 \%$ of P. aeruginosa and 20\% with RNAs from both species (Figure 2). Assuming no biases towards the formation of chimeras intra- and inter-species, we calculated the expected percentage of chimeras of each class in the experiments corresponding to the different mixtures of RNA. In this regard, it is worth considering that while $M$. pneumoniae has one rRNA operon and 32 tRNAs, $P$. aeruginosa has four rRNA operons and 63 tRNAs. For this purpose we considered the total number of fully mapped reads of each bacterium per sample. Data analysis showed that the percentage of chimeras in which the two parts corresponded to only $M$. pneumoniae, or the two species was similar to what was expected by chance (Figure 2). In the case of $P$. aeruginosa there could be a higher proportion of observed chimeras than expected. In the Cell Mix sample, there were initially many more $P$. aeruginosa cells than M. pneumoniae cells, based on the number of reads that fully mapped each species. Thus, both the expected and observed values for the chimeras of $P$. aeruginosa are much higher than in the RNA mix samples, in which the proportion of RNA was favoring M. pneumoniae (Figure 2).
All these results indicate that most chimeras observed are artifacts of the library preparation. For instance, a phenomenon named 'template switching', consisting in an exchange of templates of the reverse transcriptase, has been reported to occur whenever duplicated sequences are close to the junction site [41].

\section{Filtering criteria to discern in between real versus artifactual chimeric RNAs}

The initial analysis of the RNA-seq data described above revealed the need to create an objective criterion to discern between artifactual or real chimeric RNAs.

First, different filters were applied to the preliminary sets of SE and PE candidates to discard the potential artifacts previously reported [41]. For example, to remove the 'template switching' effect [41], all the SE candidates that presented duplicated sequences of more than $6 \mathrm{nu}$ cleotides at both sides of the junction were discarded as likely reverse transcription artifacts during the process of library preparation. Then, we grouped all SE candidates spanning the exact same junction site. In fact, a vast majority of chimeric candidates (around 80\%) was supported by only one read and were thus discarded.

Second, we considered the expression levels of the genes located at both sides of the junction. The expression values were calculated based on the fully mapped reads from the RNAseq datasets. The reason for 


\section{A. SINGLE END CANDIDATES}
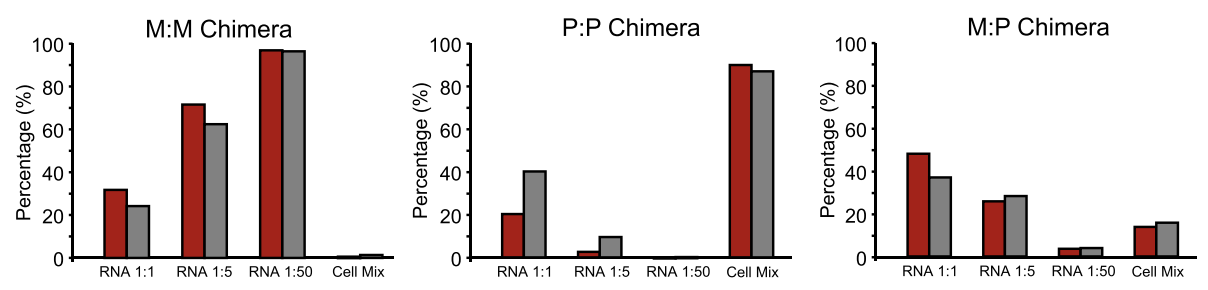

B. PAIRED-END CANDIDATES
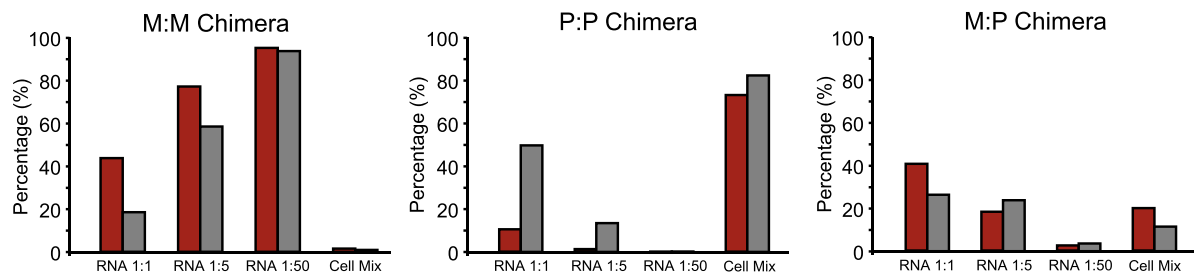

Figure 2 Identification of putative chimeric RNAs. A) Histograms show, from left to right, the percentage of different chimeric RNAs (M:M= M. pneumoniae:M. pneumoniae; $P: P=P$. aeruginosa:P. aeruginosa; $M: P=M$. pneumoniae:P. aeruginosa) obtained after single end RNAseq data analysis of the samples obtained by mixing RNAs in the different ratios $(1: 1 ; 1: 5 ; 1: 50$ and Cell mix). Grey columns indicate the percentage of expected chimeras calculated by considering the total number of reads of each species per sample and red columns indicated the percentage of obtained chimeric RNAs after RNAseq data analysis B) Histograms obtained after analyzing RNA-seq data by pair end. The data is represented following the same criteria than described for panel $\mathbf{A}$.

considering expression levels is the fact that in M. pneumoniae, there is a good correlation between the expression of a gene and the number of chimeric fragments that map inside that gene (Figure 3A) $(r=0.694)$. This correlation is much lower in $P$. aeruginosa $(r=0.267)$. The reason behind the poor correlation in P. aeruginosa, is the high abundance of ribosomal RNA in the samples (93.3\% in P. aeruginosa, and $81.2 \%$ in M. pneumoniae), which results in lower deep sequencing coverage (we obtained roughly the same number of sequencing reads for M. pneumoniae and $P$. aeruginosa, but the latter has a much larger genome and four rRNA operons). Therefore, to find a true chimera, the number of reads supporting the junction has to be significant compared to the expression of the genes forming the chimera. This issue was assessed via two confidence scores $\left(\Omega_{\mathrm{se}}\right.$ and $\Omega_{\mathrm{pe}}$, SE score and PE score; see Methods section). These scores relate the number of reads of a chimeric RNA to its expression. The least expressed gene comprising the chimera was chosen to evaluate the expression, as the limiting factor for its formation. Figure 3B represents the distribution of the scores for the 1:1 RNA mixture sample.

Additionally, it has been proposed that the pattern of the reads covering the junction may reveal the existence of possible false positive chimeras arising from PCR artifacts or errors in the mapping process [42,43]. If all reads covering a junction are identical, i.e. mapping to the exact same positions, and thus they show a box-like pattern when the coverage of the junction is represented
(Figure 3C), this chimeric RNA is likely to be an artifact. Contrarily, if the reads spanning the junction are distributed along the chimera and show a staircase-like pattern (Figure 3E and 3F), the chimera can be assumed to be real $[42,43]$. We followed two criteria that allowed us to distinguish in between these two patterns. First of all, we considered the number of unique reads (non-duplicated reads) that spanned each junction. We related this number to the total reads that mapped to that junction. Furthermore, we required that the distribution of the reads covering the same junction was uniform and thus showing a staircase pattern (see Methods). This second criteria was added when a mixed pattern was found to occur in the false positive set of chimeras $M$. pneumoniae:P. aeruginosa. In this third case a staircase-like shape is also observed, but here, one unique read likely coming from a PCR artifact is responsible for more than $50 \%$ of the total observed read counts (Figure 3D). Figure 3B depicts the chimeras passing the different filtering criteria established, representing the $\Omega_{\text {se }}$ and $\Omega_{\text {pe }}$ scores.

In order to determine which threshold to use for each of the filters applied, we used the 1:1 RNA mixture sample and we divided the fusion transcript candidates into two groups, one formed by inter-species chimeras (true false positives) and another formed by intra-species chimeras. We tested different sets of parameters and used the ones that were able to eliminate the inter-species chimeras in the final set of results. With this approach, in the end we were able to obtain a set of $29 P$. aeruginosa fusion 

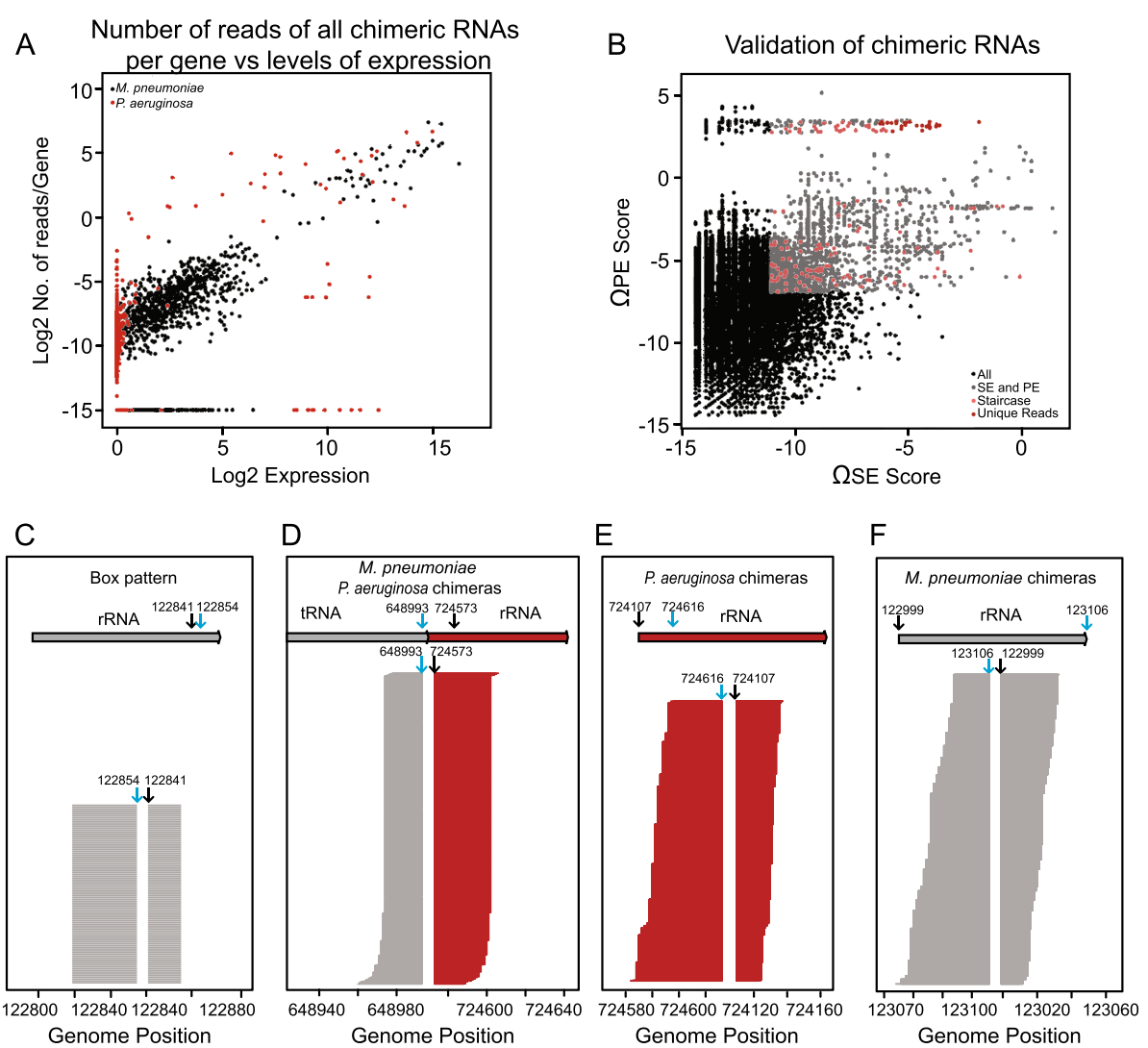

Figure 3 Validation of putative chimeric RNAs. A) Relation between levels of expression and number of chimeras. The graph represents the number of chimeric reads per gene versus gene expression levels for M. pneumoniae (in black) and P. aeruginosa (in red). Sample obtained after mixing RNA of M. pneumoniae and P. aeruginosa in a ratio 1:1. B) Representation of putative chimeric RNAs in M. pneumoniae after validation filters. Black dots represent the 43.399 initial candidates. Grey dots, putative chimeric RNAs after filtering by $\Omega_{\text {se }}$ and $\Omega_{\text {pe }}$ scores (4047). Pink dots, candidates after applying staircase filter (74). Dark red dots,the putative chimerias obtained after applying the unique reads filter (33). C) Representation of a "box-pattern" profile of the reads corresponding to a M. pneumoniae chimeria comprising two regions of the rRNA. Grey arrow represents the genomic regions of $M$. pneumoniae and blue and black arrows, the junction positions in the pileup of the sequenced reads. D) Representation of reads for an artificial chimeric RNA result of ligation of tRNA of M. pneumoniae and rRNA of P. areuginosa. Arrows represent the genomic regions of $M$. pneumoniae (in grey) and P. aeruginosa (in dark red). Blue and black arrows indicate the positions of the junction in the genome and in the pileup of the sequenced reads. E) Staircase profile can be observed for the different reads that cover the junction of the chimeric RNA in P. aeruginosa. Dark red arrow represents the genome region that codes for the RNA. Blue and black arrows indicate the positions of the junction in the genome. F) Representation of reads for a putative chimeric RNA in M. pneumoniae. Grey arrow represents the genome region that codes for the RNA. Blue and black arrows, the junction positions in the pileup of sequenced reads.

transcripts in the 1:1 RNA mixture sample that passed all the thresholds. All of these RNAs represent a circular RNA formed in the 23S rRNA. No chimeric RNAs were found in M. pneumoniae in this sample.

We applied the filtering with these same thresholds to the remaining samples. It was observed that in the RNA mixtures $1: 5$ and 1:50, in which the $P$. aeruginosa RNA concentration decreased, no chimeras appeared from this species and only few chimeras of $M$. pneumoniae were found. All putative chimeras found after the filtering are represented in Figure 4.

\section{Analysis by other software tools}

After performing strand specific paired-end RNA-seq [44], sequencing data was analyzed by three different software tools aimed to detect fusion transcripts in eukaryotic RNAseq data: FusionMap [20], ChimeraScan [25] and TopHat Fusion [26]. All these three pipelines were applied using the specified default parameters except for the maximal intron length. As no introns are present in bacterial transcriptomes, this distance was minimized in the analysis, in order to find chimeras regardless the distance in between the two parts forming them. After applying each of these pipelines to the 1:1 RNA mixture dataset, we observed large discrepancies among the results. The number of chimeras detected by the different pipelines varied largely (see Additional file 2: Table S1). Whereas TopHat Fusion found up to 350 chimeric RNAs in this sample, which were grouped into 51 sets according to their genomic location, ChimeraScan and FusionMap found more than 3000 fusion 
A

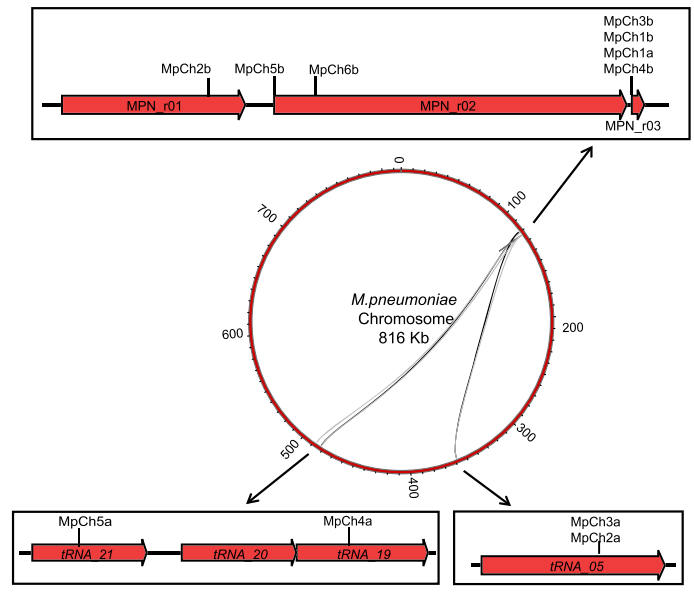

C
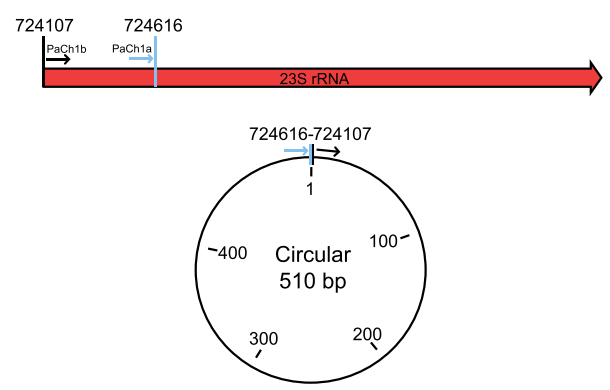

B
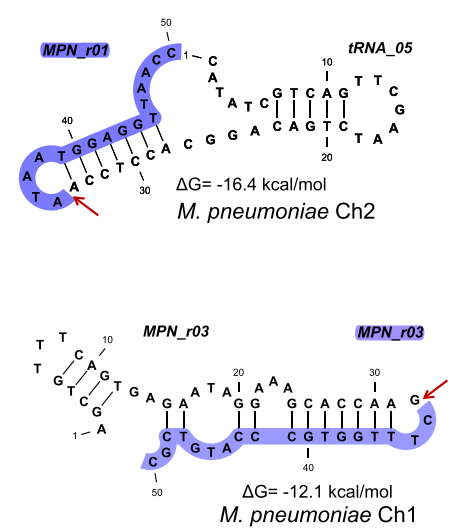

D

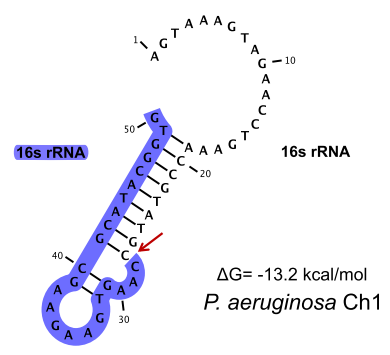

Figure 4 Putative chimeric RNAs in M. pneumoniae and P. aeruginosa. A) Circos plot representing chimeric RNAs in the genome of M. pneumoniae. Rectangles represent a zoom of the genomic region indicated with a black arrow. These regions correspond to the two parts that compound the different chimeric RNAs described in Additional file 2: Table S2 ( $a$ = region of first gene and $b=$ region of second gene). Red arrows in the rectangles indicate genes and lines in Circos plot indicate connections between genomic regions. B) Secondary structure of M. pneumoniae RNA species. Upper part of the panel shows the predicted secondary structure of a putative chimeric RNA of M. pneumoniae (MpnCh2) obtained by CLC Workbench program. Lower panel shows an example of the secondary structure for the MpnCh1, a circular RNA of M. pneumoniae. In both representations the junction is indicated with a red arrow and second part of the chimeric RNA is shadowed in blue. Complementary regions are connected with black lines. C) Circular RNA of P. aeruginosa. Circular RNA corresponding to the results described in Additional file 2: Table S3. Red arrow indicates 23 S rRNA gene, blue and black lines indicate the genome positions of the first and second regions of the circular molecule, respectively. Circular graph is a schematic representation of the obtained molecule. D) Secondary structure of the 23s rRNA chimera found in P. aeruginosa. The junction is indicated with a red arrow and second part of the chimeric RNA is shadowed in blue. Complementary regions are connected with black lines.

transcripts each, and ours only 29 , but grouped in one unique set according to their genomic location (they only were formed in the same region of the $23 \mathrm{~S}$ rRNA of $P$. aeruginosa). Furthermore, the three methods interrogated found chimeras formed by transcripts different than rRNAs and tRNAs, the most abundant ones in bacterial genomes. Whereas TopHat Fusion found 16 fusion transcripts formed by protein-coding or non-coding RNAs (4.57\% of the total chimeras detected), FusionMap found $32(0.88 \%)$ and ChimeraScan found $22(0.71 \%)$. Interestingly, as shown in the case of the 1:1 RNA mixture of both bacteria, not a single common fusion transcript was found by all three methods (Additional file 3: Figure S2A). Also, all different pipelines (except ours) found inter-species chimeras, providing proof of the presence of false positives in the results (Additional file 3: Figure S2B). These results should be regarded with caution since the three used methods were developed for analysis of eukaryotic and not prokaryotic RNAseq data.

\section{Avoiding hodgepodge in RNA-seq data}

The fact that some chimeras passed all the established thresholds does not imply that they are real RNA chimeras. In fact, no coincidences were found among the 
different protocols used to analyze the data (Additional file 2: Table S1 and Additional file 3: Figure S2A). Furthermore, chimeras found in $M$. pneumoniae were only derived from rRNAs and tRNAs, the most abundant species. However, they were not found randomly within these molecules, but they appeared to be formed in between certain 'hotspots' (Figure 4A). We observed that in many cases, these 'hotspots' corresponded to regions that showed base-pair complementarity (Figure 3B). In the case of $P$. aeruginosa the only RNA species that passed all filters using our custom pipeline in the different RNAseq experiments is a circular form of the 23S rRNA (Figure 4C) and there are $10 \mathrm{bps}$ in both sides of the junction with exact base-pair complementarity (Figure 4D). Therefore, we hypothesized that the strong secondary structure of the native molecules prevents their fully denaturalization during sample preparation using the Directional RNA-seq library preparation method (see Methods, Directional RNA-seq library preparation and sequencing). Thus, after shearing the RNA molecules by sonication, the fragments that kept hybridized could remain close in the space, favoring their ligation in the following step of the library preparation.

To test if double-stranded RNA could be behind the observed chimeras we used a different protocol (TruSeq stranded RNA-seq) to prepare the libraries for deep sequencing of the $M$. pneumoniae unmixed RNA sample (Figure 5A). In this protocol, cDNA is synthesized before the adapter ligation, and therefore the secondary structure of these molecules is removed (see Methods). After sequencing the sample prepared with this second protocol, we observed that the number of initially non-mapped reads to the reference genome decreased dramatically (from $50 \%$ to $5 \%$; Figure $5 \mathrm{~B}$ ). After performing the analysis and filtering previously described, the number of chimera candidates decreased to 137 (compared to several thousands obtained with the first protocol; see Figure 5C) and no chimeric reads were found in the final filtering steps (Figure 5C). Also, when applying the three different published pipelines stated above, no chimeras were found in any of them (See Additional file 1: Figure S1). All these data taken together suggests that the TruSeq stranded RNA-seq is the most accurate protocol to avoid artifacts in the library preparation and the described pipeline allows the filtering of artifactual chimeric RNAs. Directional RNA-seq is widely used for microRNA library preparation in eukaryotes and RNAseq in prokaryotes, which could lead to identification of non-real chimeras and/or underestimation of the expression levels of some genes.

\section{Conclusions}

Nowadays, RNA-Seq is becoming one of the most used approaches for transcriptome profiling. Different studies using this methodology have suggested the existence of chimeric RNAs and circular rRNAs in different organisms. We showed that these RNAs could be artifacts generated during library preparation, and that they are protocol-dependent. We compared two different protocols for RNA-seq library preparation and observed that theyled to very different results in terms of reported chimeric RNAs. Moreover, different sequence analysis methodologies including ours don't eliminate all nonreal chimeras. Therefore, care should be taken when selecting a protocol for library preparation and sequencing, as well as a pipeline for the analysis of the results.

\section{Methods}

\section{Bacterial strains and growth conditions}

M. pneumoniae M129 was grown in $25 \mathrm{~cm}^{2}$ tissue culture flasks with $5 \mathrm{~mL}$ of modified Hayflick medium at $37^{\circ} \mathrm{C}$ as previously described [45]. Pseudomonas aeruginosa PAO1 cells were grown at $37^{\circ} \mathrm{C}$ under agitation $(100 \mathrm{rpm})$ in $25 \mathrm{ml}$ of $7 \mathrm{H} 9$ broth medium from BD plus ADC with $0.5 \%$ glycerol and $0.05 \%$ tween 80 .

\section{RNA extractions and sample preparations}

After growing $M$. penumoniae during $96 \mathrm{~h}$ at $37^{\circ} \mathrm{C}$, cells were washed twice with PBS and lysated with $700 \mu \mathrm{l}$ of Qiazol buffer.

P. aeruginosa cells were grown during 4 days at $37^{\circ} \mathrm{C}$, after cell centrifugation the pellet was washed twice with PBS. Then, samples were lysated with $700 \mu$ l of Qiazol buffer.

RNA extractions were performed by using miRNeasy mini Kit (Qiagen) following the instructions of the manufacturer.

RNAs obtained from two bacteria were mixed in different proportions: 1:1, 1:5 and 1:50 (ratio P. aeruginosa: M. pneumoniae). These mixtures together with nonmixed wild-type RNAs were used for RNA-seq library preparation.

Also a RNA extraction was performed after mixing the cells 1:1 (M. pneumoniae:P. aeruginosa).

\section{Library preparations}

Libraries for RNA-seq were prepared following two different protocols: i) Directional RNA-seq library preparation and sequencing; ii) Illumina TruSeq stranded RNAseq sample preparation.

\section{Directional RNA-seq library preparation and sequencing}

One $\mu \mathrm{g}$ of total RNA was fragmented to around 100150 nt using NEB Next Magnesium RNA Fragmentation Module (ref. E6150S, NEB). Treatments with Antarctic phosphatase (ref. M0289S, NEB) and PNK (ref. M0201S, NEB) were performed in order to make the $5^{\prime}$ and $3^{\prime}$ ends of the RNA available for adapter ligation. Samples were further processed using the TruSeq small RNA Sample 


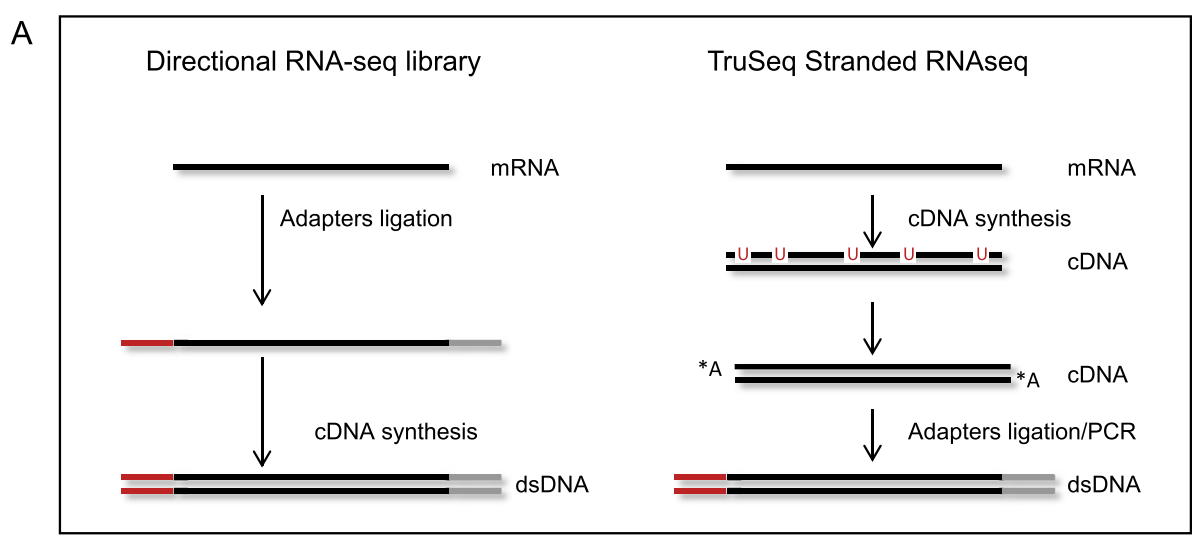

B

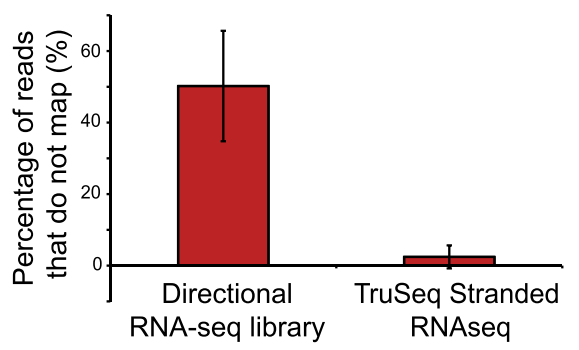

C

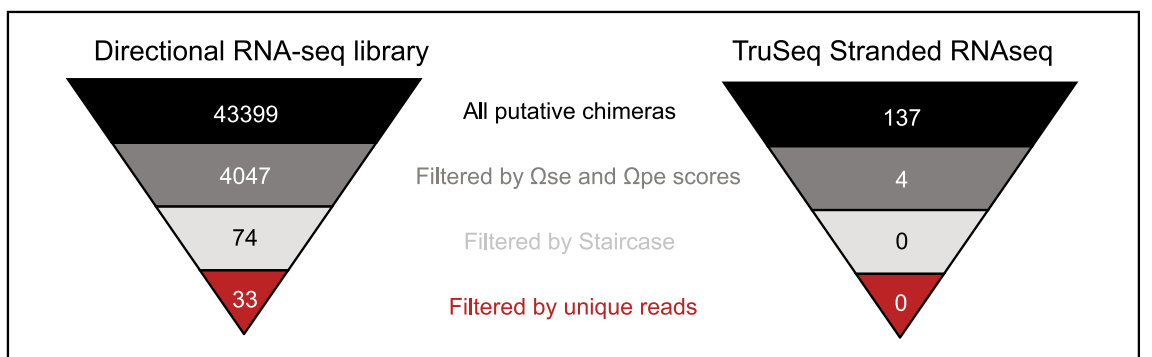

Figure 5 Comparison between different methodologies to prepare RNA-seq libraries. A) Schematic representation of the Directional RNA-seq and TruSeq Stranded RNA-seq protocols. B) Histogram representing the percentage of reads that do not map against M. pneumoniae genome in the different protocols used to prepare RNA-seq libraries. C) The inverted pyramids indicate the different number of chimeric RNAs obtained in the consecutive steps of sample validation for both protocols of library preparation.

Prep Kit (ref. RS-200-0012, Illumina) according to the manufacturer's protocol. Briefly, 3' adapters and subsequently $5^{\prime}$ adapters were ligated to the RNA. cDNA was synthesized using reverse transcriptase (SuperScript II, ref. 18064-014, Invitrogen) and a specific primer (RNA RT Primer) complementary to the $3^{\prime}$ RNA adapter. cDNA was further amplified by PCR using indexed adapters supplied in the kit. Finally, size selection of the libraries pas performed using 6\% Novex ${ }^{\oplus}$ TBE Gels (ref. EC6265BOX, Life Technologies). Fragments with insert sizes of 100 to $130 \mathrm{bp}$ were cut from the gel, and cDNA was precipitated and eluted in $10 \mu \mathrm{lEB}$.

\section{Illumina TruSeq stranded RNAseq sample preparations}

Libraries were prepared using the TruSeq Stranded mRNA Sample Prep Kit v2 (ref. RS-122-2101/2) according to the manufacturer's protocol. Briefly, $1 \mu \mathrm{g}$ of total RNA were fragmented to approximately $300 \mathrm{bp}$. cDNA was synthesized using reverse transcriptase (SuperScript II, ref. 18064-014, Invitrogen) and random primers. The second strand of the cDNA was done by removing the RNA template and synthesizes a replacement strand, incorporating dUTP in place of dTTP to generate ds cDNA. Then ds cDNA was used for library preparation. dsDNA was subjected to end repair, addition of " $A$ " bases to 3 ' ends and ligation of the barcoded Truseq adapters. All purification steps were performed using Qiagen PCR purification columns (refs. 50928106 and 50928006). Library size selection was done with $2 \%$ low-range agarose gels. Fragments with insert sizes of 200 to $400 \mathrm{bp}$ were cut from the gel, and DNA was extracted using QIAquick Gel extraction kit (ref. 50928706, Qiagen) and eluted in 
$20 \mu \mathrm{l}$ EB. Library amplification was performed by PCR on the size selected fragments using the primer cocktail supplied in the kit.

Final libraries were analyzed using Agilent DNA 1000 chip to estimate the quantity and check size distribution, and were then quantified by qPCR using the KAPA Library Quantification Kit (ref. KK4835, KapaBiosystems) prior to amplification with Illumina's cBot. Libraries were loaded at a concentration of $1.66 \mathrm{pM}$ onto the flowcell, and were paired-end sequenced, with a mate read length of 50 nts on Illumina's HiSeq 2000.

\section{RNA-seq data analysis with a custom-designed pipeline}

The workflow followed to identify the chimeric RNA candidates is shown in Figure 2. This pipeline can be divided in three sections: first, we identified the putative chimeric junctions by split-mapping the single mates of the paired-end reads. Then, the information of the paired-end reads was used to confirm the existing junctions and determine the putative chimeras. Finally, the putative chimeras were validated by using three filters: i) Confidence scores; ii) Filtering by unique reads and iii) Filtering by a staircase-like profile.

\section{Split-mapping}

For each experiment, both mates of the paired-end reads were considered as independent single-end reads. They were mapped using GEM [39] to the reference genomes of M. pneumoniae M129 or P. aeruginosa PAO1. For the experiments in which both RNAs were mixed, reads were mapped against an artificial genome formed by the union of the chromosomes of both species.

As no rRNA depletion was performed during sample preparation, the vast majority of the mapped reads correspond to the rRNAs of both bacteria. $P$. aeruginosa has four rRNA operons. Three of them were removed artificially from the reference genome, considering the remaining one as the reference rRNA operon. This was aimed not to discard all putative chimeras coming from any of them.

Reads that were not mapped in this first round were retrieved and remapped using GEM-split-mapper, a component of the GEM suite [39]. This mapper splits the input reads in two parts and tries to map both of them to different sites of the genome. Again, up to two mismatches were allowed. After this second mapping, only those reads that yielded one unique hit in the reference genome (this is, the reads that uniquely split-map to two different locations) were considered for subsequent analysis.

In order to validate the mapping of the split reads, a BLAST search of them against the reference genome was performed. Only the reads showing an agreement in between the split-mapping and the first hits of the BLAST results were retrieved and considered as singleend candidates (SE candidates; Figure 2).

\section{Paired-end mapping}

Both mates of the paired-end reads were mapped separately as single-end reads using Bowtie [38]. In this case, a full mapping of each mate was expected, with up to two mismatches. Mates were mapped to any of the reference genomes aforementioned, according to the RNA composition of each sample. After mapping both mates, they were joined to obtain the paired-end information and calculate the insert length. The insert length distribution obtained was used to determine which pairedend reads could be considered to have an abnormal insert size and thus be chimeric RNA candidates.

In addition to the reads presenting an abnormal insert length, paired-end reads in which each of the mates were mapping to different species were extracted, knowing that this set of chimeric reads will only correspond to false positive hits. Paired-end reads in which both ends were mapping to the same strand were also retrieved, as well as reads in which the second mate was mapping upstream the first mate, being candidates to represent putative circular RNAs.

\section{Candidate filtering and validation \\ Determination of confidence score for chimeric RNAs}

To discern between real and artifactual chimeric RNAs we have defined two scores $\left(\Omega_{\text {se }}\right.$ and $\left.\Omega_{\text {pe }}\right)$ based on the two different methods for the analysis (single-end, se; paired-end, pe). Each of them considers the number of reads supporting each chimeric RNA and the gene expression levels of the different mRNAs that comprise this hybrid RNA. The scores are calculated as follows:

$$
\begin{aligned}
& \Omega_{s e}=\log _{2}\left(\frac{N c_{s e}}{N_{t} / L}\right) \\
& \Omega_{p e}=\log _{2}\left(\frac{N c_{p e}}{N_{t} / L}\right)
\end{aligned}
$$

$\Omega_{\mathrm{se}}$ and $\Omega_{\mathrm{pe}}$ are the confidence scores for single-end and paired-end analysis, respectively; $\mathrm{Nc}_{\mathrm{se}}$ and $\mathrm{Nc}_{\mathrm{pe}}$ are the total number of reads that define a chimeric RNA in the different approaches performed; $\mathrm{N}_{t}$ is the total number of reads that map canonically to the gene with the lowest expression value of the chimera (which will be limiting in the chimera formation); $\mathrm{L}$ is the gene length of the least expressed gene in the chimera.

\section{Filtering by unique reads and staircase-like profile}

Here, we considered the number of unique reads (nonduplicated reads) spanning each chimeric junction. To establish a threshold, we used a score that related the number of unique sequences per chimera to the number of total reads mapping to this same chimera.

In addition to that, we also considered the distribution of the reads spanning the chimeric junction. This 
distribution should be uniform and there should not be any over-represented read, showing a staircase pattern upon coverage representation (Figure 3E, 3F). To establish a threshold for this criterion, we determined the percentage of reads corresponding the most abundant unique sequence respect to the total of reads spanning the junction.

\section{Additional files}

Additional file 1: Figure S1. Percentages of different RNA molecules. Histogram represents the percentages of different chimeric RNAs in the 1:1 ratio RNA mix sample (rRNA = ribosomal RNA; tRNA = transfer RNA; $m R N A=R N A$ of ORFs and non-coding RNAs). Pie chart represents the percentage of tRNA and rRNA that are circular or chimeric RNAs. Values next to the pie charts for the rRNA-rRNA and tRNA-tRNA bars represent the percentage of circular RNAs from the total of chimeras of these classes

Additional file 2: Table S1. Putative chimeras found by the 4 pipelines tested in two samples from different library preparation protocols. Numbers in parenthesis represent the number of chimeras clustered by genomic location (if applicable). Table S2. Mycoplasma pneumoniae chimeric RNAs obtained after validation in directional RNA-seq library preparation. Table S3. Pseudomonas aeruginosa chimeric RNAs obtained after validation in directional RNA-seq library preparation. Table S4. Putative chimeric RNAs in the 1:1 RNA mixture found by TopHat-Fusion. Table S5. Putative chimeric RNAs in the 1:1 RNA mixture found by FusionMap. Table S6. Putative chimeric RNAs in the 1:1 RNA mixture found by ChimeraScan.

Additional file 3: Figure S2. Results of the different pipelines used to analyze the data. A) Venn diagram showing the number of chimeras found by each of the pipelines used and the concordances among them. The diagram shows the values for the analysis of the 1:1 RNA mix sample, using the directional RNA-seq library preparation protocol. B) Bar plot showing the percentages of chimeras of each type found by the different pipelines tested. THF: TopHat-Fusion, CS: ChimeraScan, FM: FusionMap, OURS: Our custom designed pipeline.

\section{Abbreviations}

PE: Paired-end; SE: Single-end; THF: TopHat-Fusion; CS: ChimeraScan; FM: FusionMap; $\Omega_{\text {se: }}$ Single-end score; $\Omega_{\text {pe: }}$ Paired-end score.

\section{Competing interests}

The authors declare that they have no competing interests.

\section{Authors' contributions}

VLR carried out the cell cultures and RNA extractions, as well as performed the data analysis, and helped to draft the manuscript. LS participated in the design and coordination of the study and helped to draft the manuscript. MLS conceived of the study and participated in its design and coordination as well as drafted the manuscript. All authors read and approved the final manuscript.

\section{Acknowledgments}

The research leading to these results has received funding from the European Union Seventh Framework Programme (FP7/2007-2013), through the European Research Council, under grant agreement Nr. 232913, the Fundación Botín, the Spanish Ministry of Economy and Competitiveness (BIO2007-61762), the National Plan of R+D + i, the ISCIII -Subdirección General de Evaluación y Fomento de la Investigación- (PI10/01702), and the European Regional Development Fund (ERDF) to the ICREA Research Professor LS. We acknowledge support from the Spanish Ministry of Economy and Competitiveness, 'Centro de Excelencia Severo Ochoa 2013-2017' (SEV-2012-0208). Library preparation and sequencing was done in the CRG Genomics Unit. We thank Dr. Anna Ferrer and Dr. Heinz Himmelbauer for critical discussions and technical assistance, and Christina Kiel for critical reading and commenting the manuscript.

\section{Author details}

'EMBL/CRG Systems Biology Research Unit, Centre for Genomic Regulation (CRG), Dr. Aiguader 88, 08003 Barcelona, Spain. ${ }^{2}$ Universitat Pompeu Fabra
(UPF), Dr. Aiguader 88, 08003 Barcelona, Spain. ${ }^{3}$ Institució Catalana de Recerca i Estudis Avançats (ICREA), Pg. Lluis Companys 23, 08010 Barcelona, Spain.

Received: 25 March 2014 Accepted: 17 July 2014

Published: 29 July 2014

\section{References}

1. Park E, Williams B, Wold BJ, Mortazavi A: RNA editing in the human ENCODE RNA-seq data. Genome Res 2012, 22:1626-1633.

2. Melcher T, Maas S, Herb A, Sprengel R, Seeburg PH, Higuchi M: A mammalian RNA editing enzyme. Nature 1996, 379:460-464.

3. Schaub M, Keller W: RNA editing by adenosine deaminases generates RNA and protein diversity. Biochimie 2002, 84:791-803.

4. Djebali S, Lagarde J, Kapranov P, Lacroix V, Borel C, Mudge JM, Howald C, Foissac S, Ucla C, Chrast J, Ribeca P, Martin D, Murray RR, Yang X, Ghamsari L, Lin C, Bell I, Dumais E, Drenkow J, Tress ML, Gelpí JL, Orozco M, Valencia A, van Berkum NL, Lajoie BR, Vidal M, Stamatoyannopoulos J, Batut P, Dobin A, Harrow J, et al: Evidence for transcript networks composed of chimeric RNAs in human cells. PLOS ONE 2012, 7:e28213.

5. Gingeras TR: Implications of chimaeric non-co-linear transcripts. Nature 2009, 461:206-211.

6. Delattre O, Zucman J, Plougastel B, Desmaze C, Melot T, Peter M, Kovar H, Joubert I, de Jong P, Rouleau G, Aurias A, Thomas G: Gene fusion with an ETS DNA-binding domain caused by chromosome translocation in human tumours. Nature 1992, 359:162-165.

7. Soda M, Choi YL, Enomoto M, Takada S, Yamashita Y, Ishikawa S, Fujiwara S, Watanabe H, Kurashina K, Hatanaka H, Bando M, Ohno S, Ishikawa Y, Aburatani H, Niki T, Sohara Y, Sugiyama Y, Mano H: Identification of the transforming EML4-ALK fusion gene in non-small-cell lung cancer. Nature 2007, 448:561-566.

8. Maher CA, Kumar-Sinha C, Cao X, Kalyana-Sundaram S, Han B, Jing X, Sam L, Barrette T, Palanisamy N, Chinnaiyan AM: Transcriptome sequencing to detect gene fusions in cancer. Nature 2009, 458:97-101.

9. Sugahara J, Yachie N, Arakawa K, Tomita M: In silico screening of archaeal tRNA-encoding genes having multiple introns with bulge-helix-bulge splicing motifs. RNA 2007, 13:671-681.

10. Salgia SR, Singh SK, Gurha P, Gupta R: Two reactions of Haloferax volcanii RNA splicing enzymes: joining of exons and circularization of introns. RNA 2003, 9:319-330.

11. Randau L, Soll D: Transfer RNA genes in pieces. EMBO Rep 2008, 9:623-628.

12. Randau L: RNA processing in the minimal organism Nanoarchaeum equitans. Genome Biol 2012, 13:R63.

13. Herai RH, Yamagishi ME: Detection of human interchromosomal trans-splicing in sequence databanks. Brief Bioinform 2010, 11:198-209.

14. Kannan K, Wang L, Wang J, Ittmann MM, Li W, Yen L: Recurrent chimeric RNAs enriched in human prostate cancer identified by deep sequencing. Proc Natl Acad Sci U S A 2011, 108:9172-9177.

15. Parra G, Reymond A, Dabbouseh N, Dermitzakis ET, Castelo R, Thomson TM, Antonarakis SE, Guigo R: Tandem chimerism as a means to increase protein complexity in the human genome. Genome Res 2006, 16:37-44.

16. Akiva P, Toporik A, Edelheit S, Peretz Y, Diber A, Shemesh R, Novik A, Sorek R: Transcription-mediated gene fusion in the human genome. Genome Res 2006, 16:30-36.

17. Frenkel-Morgenstern M, Lacroix V, Ezkurdia I, Levin Y, Gabashvili A, Prilusky J, Del Pozo A, Tress M, Johnson R, Guigo R, Valencia A: Chimeras taking shape: potential functions of proteins encoded by chimeric RNA transcripts. Genome Res 2012, 22:1231-1242.

18. Frenkel-Morgenstern $M$, Valencia A: Novel domain combinations in proteins encoded by chimeric transcripts. Bioinformatics 2012, 28:i67-i74

19. Francis RW, Thompson-Wicking K, Carter KW, Anderson D, Kees UR, Beesley AH: FusionFinder: a software tool to identify expressed gene fusion candidates from RNA-Seq data. PLOS ONE 2012, 7:e39987.

20. Ge H, Liu K, Juan T, Fang F, Newman M, Hoeck W: FusionMap: detecting fusion genes from next-generation sequencing data at base-pair resolution. Bioinformatics 2011, 27:1922-1928.

21. Liu C, Ma J, Chang CJ, Zhou X: FusionQ: a novel approach for gene fusion detection and quantification from paired-end RNA-Seq. BMC bioinformatics 2013, 14:193.

22. Li Y, Chien J, Smith DI, Ma J: FusionHunter: identifying fusion transcripts in cancer using paired-end RNA-seq. Bioinformatics 2011, 27:1708-1710. 
23. Wang $K$, Singh D, Zeng Z, Coleman SJ, Huang Y, Savich GL, He X, Mieczkowski P, Grimm SA, Perou CM, MacLeod JN, Chiang DY, Prins JF, Liu J: MapSplice: accurate mapping of RNA-seq reads for splice junction discovery. Nucleic Acids Res 2010, 38:e178.

24. Dobin A, Davis CA, Schlesinger F, Drenkow J, Zaleski C, Jha S, Batut P, Chaisson M, Gingeras TR: STAR: ultrafast universal RNA-seq aligner. Bioinformatics 2013, 29:15-21

25. Iyer MK, Chinnaiyan AM, Maher CA: ChimeraScan: a tool for identifying chimeric transcription in sequencing data. Bioinformatics 2011, 27:2903-2904.

26. Kim D, Salzberg SL: TopHat-Fusion: an algorithm for discovery of novel fusion transcripts. Genome Biol 2011, 12:R72

27. Haas BJ, Gevers D, Earl AM, Feldgarden M, Ward DV, Giannoukos G, Ciulla D, Tabbaa D, Highlander SK, Sodergren E, Methé B, DeSantis TZ, The Human Microbiome Consortium, Petrosino JF, Knight R, Birren BW: Chimeric 16S rRNA sequence formation and detection in Sanger and 454-pyrosequenced PCR amplicons. Genome Res 2011, 21:494-504.

28. Doose G, Alexis M, Kirsch R, Findeiss S, Langenberger D, Machne R, Morl M, Hoffmann S, Stadler PF: Mapping the RNA-Seq trash bin: unusual transcripts in prokaryotic transcriptome sequencing data. RNA Biol 2013 10:1204-1210.

29. Himmelreich R, Hilbert H, Plagens H, Pirkl E, Li BC, Herrmann R: Complete sequence analysis of the genome of the bacterium Mycoplasma pneumoniae. Nucleic Acids Res 1996, 24:4420-4449.

30. Stover CK, Pham XQ, Erwin AL, Mizoguchi SD, Warrener P, Hickey MJ, Brinkman FS, Hufnagle WO, Kowalik DJ, Lagrou M, Garber RL, Goltry L, Tolentino E, Westbrock-Wadman S, Yuan Y, Brody LL, Coulter SN, Folger KR, Kas A, Larbig K, Lim R, Smith K, Spencer D, Wong GKS, Wu Z, Paulsen IT, Reizer J, Saier MH, Hancock REW, Lory S, et al: Complete genome sequence of Pseudomonas aeruginosa PAO1, an opportunistic pathogen. Nature 2000, 406:959-964.

31. Guell M, van Noort V, Yus E, Chen WH, Leigh-Bell J, Michalodimitrakis K, Yamada T, Arumugam M, Doerks T, Kuhner S, Rode M, Suyama M, Schmidt S, Gavin AC, Bork P, Serrano L: Transcriptome complexity in a genome-reduced bacterium. Science 2009, 326:1268-1271.

32. Jima DD, Zhang J, Jacobs C, Richards KL, Dunphy CH, Choi WW, Au WY Srivastava G, Czader MB, Rizzieri DA, Lagoo AS, Lugar PL, Mann KP, Flowers CR, Bernal-Mizrachi L, Naresh KN, Evens AM, Gordon LI, Luftig M, Friedman DR, Weinberg JB, Thompson MA, Gill Jl, Liu Q, How T, Grubor V, Gao Y, Patel A, Wu H, Zhu J, et al: Deep sequencing of the small RNA transcriptome of normal and malignant human $B$ cells identifies hundreds of novel microRNAs. Blood 2010, 116:e118-e127.

33. Pfeffer $\mathrm{S}$, Lagos-Quintana M, Tuschl T: Cloning of small RNA molecules. Curr Protoc Mol Biol 2005, Chapter 26:Unit 2624

34. Li Z, Zhang Z, Yan P, Huang S, Fei Z, Lin K: RNA-Seq improves annotation of protein-coding genes in the cucumber genome. BMC Genomics 2011, 12:540.

35. Mercer TR, Gerhardt DJ, Dinger ME, Crawford J, Trapnell C, Jeddeloh JA, Mattick JS, Rinn JL: Targeted RNA sequencing reveals the deep complexity of the human transcriptome. Nat Biotechnol 2012, 30:99-104.

36. Li S, Dong X, Su Z: Directional RNA-seq reveals highly complex condition-dependent transcriptomes in E. coli K12 through accurate full-length transcripts assembling. BMC Genomics 2013, 14:520.

37. Perkins TT, Kingsley RA, Fookes MC, Gardner PP, James KD, Yu L, Assefa SA, He M, Croucher NJ, Pickard DJ, Maskell DJ, Parkhill J, Choudhary J, Thomson NR, Dougan G: A strand-specific RNA-Seq analysis of the transcriptome of the typhoid bacillus Salmonella typhi. PLoS Genet 2009, 5:e1000569.

38. Langmead B, Trapnell C, Pop M, Salzberg SL: Ultrafast and memoryefficient alignment of short DNA sequences to the human genome. Genome Biol 2009, 10:R25.

39. Marco-Sola S, Sammeth M, Guigo R, Ribeca P: The GEM mapper: fast, accurate and versatile alignment by filtration. Nat Methods 2012, 9:1185-1188.

40. Kircher M, Sawyer S, Meyer M: Double indexing overcomes inaccuracies in multiplex sequencing on the Illumina platform. Nucleic Acids Res 2012, 40:e3.

41. Cocquet J, Chong A, Zhang G, Veitia RA: Reverse transcriptase template switching and false alternative transcripts. Genomics 2006, 88:127-131.

42. Edgren H, Murumagi A, Kangaspeska S, Nicorici D, Hongisto V, Kleivi K, Rye $\mathrm{H}$, Nyberg S, Wolf M, Borresen-Dale AL, Kallioniemi O: Identification of fusion genes in breast cancer by paired-end RNA-sequencing. Genome Biol 2011, 12:R6.
43. Abate F, Acquaviva A, Paciello G, Foti C, Ficarra E, Ferrarini A, Delledonne M, lacobucci I, Soverini S, Martinelli G, Macii E: Bellerophontes: an RNA-Seq data analysis framework for chimeric transcripts discovery based on accurate fusion model. Bioinformatics 2012, 28:2114-2121.

44. Vivancos AP, Guell M, Dohm JC, Serrano L, Himmelbauer H: Strand-specific deep sequencing of the transcriptome. Genome Res 2010, 20:989-999.

45. Yus E, Guell M, Vivancos AP, Chen WH, Lluch-Senar M, Delgado J, Claude Gavin A, Bork P, Serrano L: Transcription start site associated RNAs in bacteria. Mol Syst Biol 2012, 8:585.

doi:10.1186/1471-2164-15-633

Cite this article as: Lloréns-Rico et al:: Assessing the hodgepodge of non-mapped reads in bacterial transcriptomes: real or artifactual RNA chimeras? BMC Genomics 2014 15:633.

\section{Submit your next manuscript to BioMed Central and take full advantage of:}

- Convenient online submission

- Thorough peer review

- No space constraints or color figure charges

- Immediate publication on acceptance

- Inclusion in PubMed, CAS, Scopus and Google Scholar

- Research which is freely available for redistribution

Submit your manuscript at www.biomedcentral.com/submit
C) Biomed Central 